\title{
Value of prostate gland volume measurement by transrectal US in prediction of the severity of lower urinary tract symptoms
}

\author{
Idil Gunes Tatar, Onur Ergun, Pinar Celtikci, Erdem Birgi, Baki Hekimoglu
}

Diskapi Yildirim Beyazit Training and Research Hospital, Department of Radiology, Ankara, Turkey

\begin{abstract}
Aim: The aim of our study was to analyze the value of prostate gland volume measurement by transrectal ultrasonography (TRUS) in prediction of severity of lower urinary tract symptoms (LUTS). Material and methods: One hundred and one patients undergoing TRUS guided core needle prostate biopsy for the investigation of prostate cancer were prospectively evaluated. All patients had filled in the international prostate symptom score (IPSS) scala, has been tested for PSA and prostate volume measurement was done by TRUS prior to biopsy. According to their biopsy results, patients were divided into two groups: negative and positive for malignancy. Results: There was a significant but low correlation of 0.37 between IPSS and prostate volume measurement by TRUS ( $\mathrm{p}=0.001$ ). Serum free prostate specific antigen (fPSA), total PSA (tPSA), ratio of fPSA to tPSA and PSA density did not show a statistically significant correlation with IPSS ( $>0.05$ ). No statistically significant difference in IPSS between the benign and malignant groups $(\mathrm{p}=0.681)$ was found. In the benign group, mean IPSS was $14.59 \pm 8.73$, range: 0 -35 while in the malignant group, mean IPSS was $15.33 \pm 9.22$, range: $3-28$. Conclusions: Prostate volume measurement by TRUS is a poor predictor for the determination of the severity of LUTS therefore IPSS should be primarily considered for the determination of the severity of LUTS. Also, according to this study conducted with patients who were at their first set of prostate biopsy, IPSS cannot be used as a predictor of malignancy. PSA values were not related to IPSS.

Keywords: prostate volume, transrectal ultrasonography, international prostate symptom score, lower urinary tract symptoms
\end{abstract}

\section{Introduction}

Prostatic enlargement resulting in benign prostatic hyperplasia (BPH) is a chronic, progressive disease which is common in male population mostly after the sixth decade [1]. BPH presents with lower urinary tract symptoms (LUTS) related to voiding (slow stream, splitting or spraying, intermittency, hesitancy, straining and terminal dribble), storage (increased daytime frequency, nocturia, urgency and urinary incontinence), and post-micturition (feeling of incomplete emptying, post-micturition dribble) consequently decreasing the quality of life [2].

Received 19.06.2014 Accepted 19.08.2014

Med Ultrason

2014, Vol. 16, No 4, 315-318

Corresponding author: Idil Gunes Tatar, M.D.

12 Irfan Bastug Street

06110, Diskapi-Altındag/ Ankara - TURKEY

Phone:+90 3125962616

Email: idil.gunes.tatar@gmail.com
The initial diagnostic challenge in patients presenting with LUTS is to establish that the symptoms are due to $\mathrm{BPH}$. Then the secondary aim is to determine the severity of BPH [3]. In the evaluation of BPH determination of the symptom score, medical history, digital rectal examination, urinalysis, serum prostate specific antigen (PSA) measurement are suggested for men over 50 years old. Urinary cytologies, transrectal ultrasonography (TRUS) measurement of the prostate, uroflowmetry, postvoid residual urine volume analysis, pressure-flow urodynamics, and filling cystometry are additional examinations which can be performed with a specific purpose such as to confirm the diagnosis, to determine the severity of $\mathrm{BPH}$, or to choose treatment $[4,5]$.

The severity of LUTS can be evaluated by the American Urological Association Symptom Score (AUASS) or the International Prostate Symptom Score (IPSS). AUASS is a scala which consists of seven questions involving LUTS related to voiding and storage, IPSS incorporates one additional question about the affect of LUTS 
on the quality of life [6-8]. Enlargement of prostate volume is associated with aggravation of LUTS, acute urinary retention and increased need for BPH surgery [6]. Prostatic volume assessment is therefore considered to be useful to determine treatment options and outcomes $[7,9,10]$. Measurement of prostate volume using ultrasonography (US) comprises different approaches, mainly transrectal and sometimes transabdominal. Transrectal route is reported to be the most accurate method [11,12].

In this research we aimed to analyze the value of prostate gland volume measurement by TRUS in prediction of the severity of LUTS due to the fact that there are only a few studies analyzing this correlation in the English literature.

\section{Material and methods}

Following the approval of the local Ethics Committee of our institution, from August 2013 to January 2014, 113 consecutive patients referred to our Radiology Department by the Urology Department for TRUS guided core needle prostate biopsy for the exclusion of malignancy were considered for this study.

\section{Study group}

Among the 113 men above the age of 50 referred for TRUS guided core needle prostate biopsy to exclude malignancy, 5 patients were excluded due to inappropriate International Normalized Ratio (INR) value ( $>1.4)$ and 7 patients due to the history of previously known prostate malignancy and/or previous radiation therapy. None of the patients had other possible causes of LUTS such as metabolic disorders (diabetes mellitus, nephrogenic type diabetes insipidus), neurologic disorders (multiple sclerosis, cerebrovascular events, lumbosacral disc diseases) or concomitant use of pharmacological agents (diuretics, alpha-agonists, anticholinergics) according to the prior detailed medical history. The remaining 101 patients constituted the study cohort. All patients were informed about the study and gave their written consent

\section{Serum analysis}

Serum samples were obtained a week before prostate biopsy. Free PSA (fPSA) and total PSA (tPSA) values were measured by chemiluminescence immunoassay kit in the biochemistry department of our institution. The reference range of free PSA was accepted as $0-3.3 \mathrm{ng} / \mathrm{mL}$ and total PSA was accepted as $0-3.9 \mathrm{ng} / \mathrm{mL}$. Ratio of fPSA to tPSA and PSA density which is the ratio of total PSA to prostate volume was later calculated for each patient.

\section{IPSS}

Prior to the biopsy all patients included in the study filled in the IPSS scale (seven questions related to LUTS questioning the severity of incomplete emptying, fre- quency, intermittency, urgency, weak stream, straining and nicturia). The answers were assigned points from 0 to 5 increasing with the severity of the symptoms. The total score therefore ranged between 0 to 35 . There was also one question for the evaluation of the quality of life which was not included in the calculation of the total score. Categorization of LUTS symptoms were interpreted as mild (symptom score $\leq 7$ ), moderate (symptom score range between 8 to 19) and severe (symptom score range between 20 to 35).

\section{TRUS and biopsy}

TRUS evaluation and volume measurement of the prostate gland followed by TRUS guided core biopsy were performed in all patients by two radiologists with 12 and 10 years of sonography experience in consensus using Logiq S6, GE Healthcare, Little Chalfont, United Kingdom. Prostate volume was measured using the prolate ellipse formula (height $\mathrm{x}$ width $\mathrm{x}$ length $\mathrm{x} \pi / 6$ ) [13]. Six core biopsy materials were obtained from each lobe covering all segments, making a total of 12 biopsies for each patient. An automatic core biopsy device (Bio-Pince ${ }^{\circledR}$ Full-Core Biopsy Instruments; Surgical Specialties Co. (Angiotech), Vancouver, Canada) with a $18 \mathrm{G}(1.2 \mathrm{~mm}) \times 20 \mathrm{~cm}$ needle, adjusted to 13 or $23 \mathrm{~mm}$ stroke length, according to the depth of the prostate gland, was used for the biopsy procedure.

\section{Pathology}

Biopsy specimens were fixated and transferred in $10 \%$ formaldehyde solution. Tissue samples were embedded in paraffin and routine hematoxylin-eosin staining was applied to section samples. If necessary, immunohistochemical staining was applied. According to the pathological evaluation of the TRUS guided biopsy samples, patients were divided into two groups: negative and positive for malignancy.

\section{Statistical analysis}

Statistical analysis were performed by statistics software; SPSS 16 (Chicago, USA). Independent Sample $\mathrm{T}$-Test was used to analyze the presence of a difference in age, height and body-mass index (BMI) between the groups negative and positive for malignancy. To analyze the presence of a difference in weight, prostate volume and IPSS between the groups negative and positive for malignancy Mann-Whitney U test was used. Spearman correlation coefficient analysis was done for the correlation between age, weight, height, BMI, prostate volume and IPSS as well as between age, weight, height, BMI and prostate volume. $\mathrm{P}$ values less than 0.05 were considered to be statistically significant.

\section{Results}

The patient characteristics of our study group are presented in table I. 
Table I. Descriptive characteristics of the study sample.

\begin{tabular}{lc}
\hline Characteristic & \\
\hline Age $($ year $)$ & $62.88 \pm 8.26$ \\
Height $(\mathrm{cm})$ & $169.35 \pm 6.79$ \\
Body weight $(\mathrm{kg})$ & $78.05 \pm 12.67$ \\
Body mass index $\left(\mathrm{kg} / \mathrm{m}^{2}\right)$ & $26.98 \pm 4.12$ \\
Prostate Volume $(\mathrm{mL})$ & $53.87 \pm 26.15$ \\
IPSS* & 17.30 \\
\hline
\end{tabular}

Data are expressed in mean \pm standard deviation; * International Prostate Symptom Score

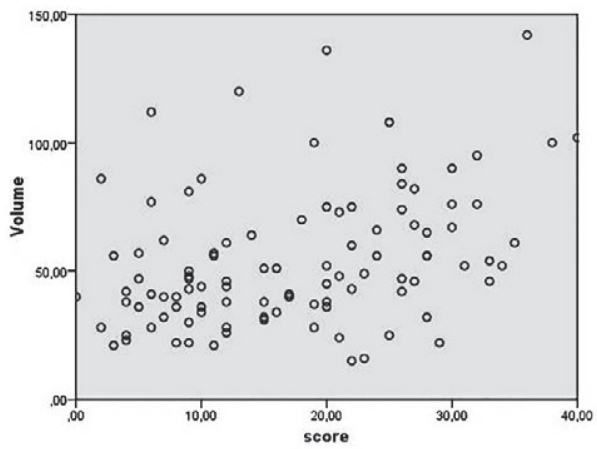

Fig 1. Correlation between international prostate symptom score and prostate volume.

In the whole group there was a low positive correlation between age with IPSS $(0.24, \mathrm{p}=0.05$ and 0.23 respectively, $\mathrm{p}=0.05$ ) and a low negative correlation between height and IPSS $(0.20, \mathrm{p}=0.05)$. Weight and BMI did not show a statistically significant correlation with IPSS ( $>0.05)$. There was a statistically significant but low correlation of 0.37 between IPSS and prostate volume ( $\mathrm{p}=0.001$ ) (fig 1). There was a low positive correlation between BMI and weight with prostate volume $(0.34$ and 0.27 respectively, $p=0.05$ ). Age and height did not show a statistically significant correlation with prostate volume ( $\mathrm{p}>0.05)$.

After histopathological examination 15 patients were found positive for malignancy. The comparison between the 2 groups (negative and positive for malignancy) is detailed in table II.

\section{Discussions}

According to the results of this study there was a statistically significant correlation between prostate volume measurement by TRUS and IPSS. This means that the patients with larger prostate volume have higher IPSS, hence experience more severe LUTS. But the low Spearman correlation $(0.37)$ between prostate volume
Table II. Characteristics of the patients negative and positive for malignancy.

\begin{tabular}{lccc}
\hline & \multicolumn{3}{c}{ Patients $(\mathrm{n}=101)$} \\
& $\begin{array}{c}\text { negative for } \\
\text { malignancy } \\
(\mathrm{n}=86)\end{array}$ & $\begin{array}{c}\text { positive for } \\
\text { malignancy } \\
(\mathrm{n}=15)\end{array}$ & $\begin{array}{c}\mathrm{p} \\
\text { value }\end{array}$ \\
\hline Free PSA $(\mathrm{ng} / \mathrm{mL})$ & $2.47 \pm 4.15$ & $13.57 \pm 17.84$ & 0.001 \\
Total PSA $(\mathrm{ng} / \mathrm{mL})$ & $8.23 \pm 9.61$ & $36.11 \pm 54.80$ & 0.001 \\
Ratio of fPSA to tPSA & $0.39 \pm 0.78$ & $0.48 \pm 0.36$ & 0.188 \\
PSA density $\left(\mathrm{ng} / \mathrm{mL} / \mathrm{cm}^{3}\right)$ & $0.16 \pm 0.15$ & $0.95 \pm 1.42$ & 0.000 \\
IPSS & $14.59 \pm 8.73$ & $15.33 \pm 9.22$ & 0.681 \\
\hline
\end{tabular}

Data are expressed in mean \pm standard deviation; n-number of patients; IPSS-International Prostate Symptom Score; PSA- prostate specific antigen

and IPSS showed that the reliability of prostate volume measurement by TRUS seems to be questionable for the determination of the severity of LUTS.

Sciarra et al [14] evaluated 400 men with LUTS and investigated the presence of difference in age, IPSS and prostate volume in 543 patients with and without benign prostatic hyperplasia. Authors reported that IPSS was weakly correlated with age and prostate volume. They concluded that the weak correlation was a result of their study sample which was selected among the patients from the urology clinic [14]. Eckhardt et al [15] also carried out a study which investigated the difference in IPSS, prostate volume and urodynamic findings in male volunteers with and without LUTS and in patients with LUTS suggestive of BPH. Their results indicated that volunteers without LUTS had smaller prostate volumes, less residual volume, lower symptom scores, a lower urethral resistance factor, a higher maximal free flow rate and a higher effective capacity than volunteers with LUTS. These variables were closely similar in volunteers with LUTS to those of the patients with LUTS except prostate volume being smaller in the former group [15]. In another prospective study including 120 patients Udeh et al analyzed the correlation between pre-treatment IPSS in BPH patients and prostate volume measured by transabdominal US. Authors did not find a significant correlation between IPSS and prostate volume [16]. Aktas et al investigated the effect of prostate volume on voiding function and IPSS score deterioration after TRUS guided prostate biopsy. Authors concluded that patients with prostate volume greater than $38.8 \mathrm{~mL}$ were more likely to have decreased IPSS scores following TRUS guided prostate biopsy [17].

Concerning the relation between IPSS and prostate volume, our results were in accordance with the study of Sciarra et al but remotely coherent with the study of Aktas 
et al We believe the reason might be our study sampling involving urology clinic based population such as Sciarra et al. On the other hand, a discordance arose between our results and the study of Udeh et al which can be attributed to the different methods used for the estimation of the prostate volume. Udeh et al utilized transabdominal US where as we utilized TRUS in our study, which is considered to be more accurate for determining the prostate volume. Thus we believe that our results are more dependable regarding the correlation between IPSS and prostate volume.

Our study has some limitations. The study group was limited in number and included only patients with LUTS from the urology clinic. Further investigations with study cohorts including non-urological patient groups are required for analyzing the correlation between IPSS and prostate.

\section{Conclusions}

Prostate volume measurement by TRUS is a poor predictor for the determination of the severity of LUTS and IPSS should be primarily considered for the determination of the severity of LUTS. On the other hand according to this study conducted with patients who were at their first set of prostate biopsy, IPSS cannot be used as a predictor of malignancy. PSA values are not related to IPSS.

\section{Conflict of interest: none}

\section{References}

1. Emberton M, Andriole GL, de la Rosette J, et al. Benign prostatic hyperplasia: a progressive disease of aging men. Urology 2003; 61: 267-273.

2. Abrams P, Cardozo L, Fall M, et al. The standardisation of terminology of lower urinary tract function: Report from the Standardisation Sub-committee of the International Continence Society. Neurourol Urodyn 2002; 21: 167-178.

3. Lepor H. Evaluating men with benign prostatic hyperplasia. Rev Urol 2004; 6 Suppl 1: S8-S15.

4. McConnell JD, Barry MJ, Bruskewitz RC, et al. Rockville, Md: Agency for Health Care Policy and Research; 1994. Benign prostatic hyperplasia: diagnosis and treatment. Clinical Practice Guideline No 8. AHCPR. Publication 94-0582.

5. Denis L, Griffiths K, Khoury S, et al. Editors. Proceedings of the 4th International Consultation on BPH. Plymouth, UK: Health Publication Ltd; 1998.
6. Jiang YH, Lin VC, Liao CH, Kuo HC. International Prostatic Symptom Score-voiding/storage subscore ratio in association with total prostatic volume and maximum flow rate is diagnostic of bladder outlet-related lower urinary tract dysfunction in men with lower urinary tract symptoms. PLoS One 2013; 8: e59176.

7. Oelke M, Bachmann A, Descazeaud A, et al. EAU guidelines on the treatment and follow-up of non-neurogenic male lower urinary tract symptoms including benign prostatic obstruction. Eur Urol 2013; 64: 118-140.

8. Barry MJ, Fowler FJ Jr, O'Leary MP, et al. The American Urological Association symptom index for benign prostatic hyperplasia. The Measurement Committee of the American Urological Association. J Urol 1992; 148: 1549-1557.

9. Jacobsen SJ, Jacobsen DJ, Girman CJ, et al. Natural history of prostatism: Risk factors for acute urinary retention. J Urol 1997; 158: 481-487.

10. Marberger MJ, Andersen JT, Nickel JC, et al. Prostate volume and serum prostate-specific antigen as predictors of acute urinary retention. Combined experience from three large multinational placebo-controlled trials. Eur Urol 2000; 38: 563-568.

11. Wadanabe H, Igari D, Tanahashi Y, Harada K, Saito M. Measurements of size and weight of prostate by means of transrectal ultrasonotomography. Tohoku J Exp Med 1974; 114: 277-285.

12. Hastak SM, Gammelgaard J, Holm HH. Transrectal ultrasonic volume determination of the prostate-preoperative and postoperative study. J Urol 1982; 127: 1115-1118.

13. Park SB, Kim JK, Choi SH, Noh HN, Ji EK, Cho KS. Prostate volume measurement by TRUS using heights obtained by transaxial and midsagittal scanning: comparison with specimen volume following radical prostatectomy. Korean J Radiol 2000; 1: 110-113.

14. Sciarra A, D’Eramo G, Casale P, et al. Relationship among symptom score, prostate volume, and urinary flow rates in 543 patients with and without benign prostatic hyperplasia. Prostate 1998; 34: 121-128.

15. Eckhardt MD, van Venrooij GE, Boon TA. Symptoms, prostate volume, and urodynamic findings in elderly male volunteers without and with LUTS and in patients with LUTS suggestive of benign prostatic hyperplasia. Urology 2001; 58: 966-971.

16. Udeh EI, Ozoemena OF, Ogwuche E. The relationship between prostate volume and international prostate symptom score in Africans with benign prostatic hyperplasia. Niger J Med 2012; 21: 290-295.

17. Aktas BK, Bulut S, Gokkaya CS, et al. Association of prostate volume with voiding impairment and deterioration in quality of life after prostate biopsy. Urology 2014; 83: 617621. 